\title{
Prevalence of Haemoptysis in PCR Positive Covid-19 Patients with Sars Covid-2 Pneumonia
}

\author{
SABO, Ahmed Mohammed ${ }^{1} \quad$ IBRAHIM, Usman Kusfa ${ }^{2}$ \\ Galam, Nanyak Zingfa ${ }^{1}$ \\ 1.Department of Human Physiology, Faculty of Basic Medical Sciences, \\ College of Health Sciences, University Of Jos \\ 2.Department of Haematology and Blood Transfusion, \\ Ahmadu Bello University Teaching Hospital (ABUTH) Shika, Zaria. Nigeria
}

\begin{abstract}
Covid-19 belongs to the group of corona viruses, morphologically shaped as crown with spikes at the rim, the viral spikes being made up of protein $\mathrm{S}_{\mathbf{1}}$ and $\mathrm{S}_{\mathbf{2}}$. Apart from the ability of SARS Covid-2 (Covid-19) to induce massive inflammation in the lungs of infected patients, an additional compounding factor that accompanies the cytokine storm is thrombo-embolic phenomenon which has been demonstrated in a number of autopsies/ Haemoptysis as a symptom is considered outcome at an outcome of the clotting and haemorrhagic phenomenon. The aim of this work is to establish the prevalence of haemoptysis in covid-19 pneumonia as a possible consequence of the combined damaging effect of cytokine storm and the thrombo-embolic phenomenon. One hundred and twenty Covid-19 patients were selected through record after obtaining ethical clearance/ In no particular order and sticking only to random sampling, study group were picked from northeast, northwest and north central geopolitical zones of Nigeria. Medical record of each is sorted by by care givers to specifically evaluate the presence of the symptom of haemoptysis at the latter and recovery stage after the sneezing, anosmia and itchy throat early stage and all through the stage where there is cough and deep retrosternal pain in response to the cough with accompanying difficulty in breathing. The result indicates the presence of haemoptysis in 10\% of PCR Covid-19 confirmed cases of pneumonia.
\end{abstract}

Keywords: PCR, Covid-19, SARS Covid-2, Pneumonia, Haemoptysis,

DOI: $10.7176 / \mathrm{JHMN} / 90-02$

Publication date:June $30^{\text {th }} 2021$

\section{INTRODUCTION}

Among the flu causing viruses, like the influenza viruses, the corona viruses have for long been entertained as candidates that may possibly assume global spread, a pandemic that may shock the world unlike anything that has ever been seen. The covid-19 pandemic, prediction could be said to have come to pass. Even Hollywood movie makers for decades have borrowed leaf from these predictions of viral holocaust and made blockbusters that have largely affected public opinion.

What biomedical scientists are postulating is that, this covid-19 pandemic may be the first but certainly not the last. More zoonotic attacks and the mutations of the current SARS Covid-2 are frightening prospect and realities of the day.

Certain viruses are known to have the ability to induce bleeding in diseases and can mostly be classified as hemorrhagic fever viruses. Such diseases include lassa fever, Ebola etc. while covid-19 does not belong to this category of viruses causing bleeding, the SARS Covid-2 virus has been shown to cause massive and a damaging inflammatory reaction through a phenomenon recognized as cytokine storm compounded by the thrombo-embolic phenomenon.

Inflammation is the reaction to injury of the vascular and supporting elements of a living tissue and it is characterize by the outpouring of protein-rich exudates. Inflammation functions to limit injury and commences the process of healing. Overwhelming inflammation can be counterproductive as they may cause dysfunction and exacerbate further injury, thus in principle warranting the use of anti-inflammatory agents for treatment. Cytokine storm has been proposed as one of the mechanism by which Covid-19 phenomenon causes huge damage to the lungs and compromise oxygen uptake thus lower percent oxygen saturation of the blood. Hypoxia eventually leads to debilitation and death. The ventilation perfusion coefficient gets elevated markedly as a proof. Thromboembolic phenomenon compromises perfusion of the alveoli and is thought be a factor, in part, of the process involved in damages and extravasation of blood that may reflect as haemoptysis.

\section{AIM AND OBJECTIVE}

To determine the percentage of PCR Covid-19 +ve patients with SARS Covid-2 pneumonia who have haemoptysis as a symptom or sign

To determine the percentage of those with described earlier with haemoptysis haemoptysis who are over 50 years of age and above and those who are less than 50 years. 


\section{MATERIAL AND METHOD}

One hundred and twenty patients suffering from covid-19 were selected using stratified random sampling method, 20 each from Nigeria's six geopolitical zones, Northeast, Northwest, North Central, Southeast, South West and South-South. The age range for those recruited into the study is from 18 to 80 years The medical information from the different approved centers for treatment of Covid-19 were sourced despite the lack of clear cut official policy on biomedical research on Covid-19 and the lack of standby research office dedicated to Covid-19 research related activities. The fear, uncertainty, lockdowns and lack of containment facility in virology laboratories have been reported to have largely paralyzed research efforts. The medical information obtained for this research was simplistic, no name and no center number included. Only the age of the patients, the onset of the symptoms and the presence or absence of haemoptysis were recorded along with the percent Oxygen Saturation of Blood measured for the patients and the time of its onset of the disease estimated from the time of the initial symptoms. TABLE 1

\section{TABLE OF DISTRIBUTION OF SOME COVID-19 MEDICAL FEATURES WITH PERCENTAGES

\begin{tabular}{lll}
\hline $\mathrm{S} / \mathrm{N}$ & $\begin{array}{l}\text { Total } \\
\text { number }\end{array}$ & Percentages $(\%)$ \\
\hline 1 & No of PCR+ve Covid-19 patients & \\
\hline
\end{tabular}

1 No of PCR+ve Covid-19 patients with SARS 120
Covid-2 Pneumonia

2 No of PCR + ve Covid-19 patients with SARS 22 Covid-2 Pneumonia and Haemoptysis

3 Number of PCR ve+ Covid-19 Patients with SARS 98 Covid-2 Pneumonia without Haemoptysis

4 PCR +ve Covid-19 Patients with SARS Covid-2 Pneumonia that have Haemoptysis who are 50 years and above

5 PCR +ve Covid-19 Patients with SARS covid-2 5 Pneumonia that have Haemoptysis who are below 50 years

\section{BAR CHART SHOWING THE DISTRIBUTION OF SOME COVID-19 MEDICAL FEATURES WITH PERCENTAGES}

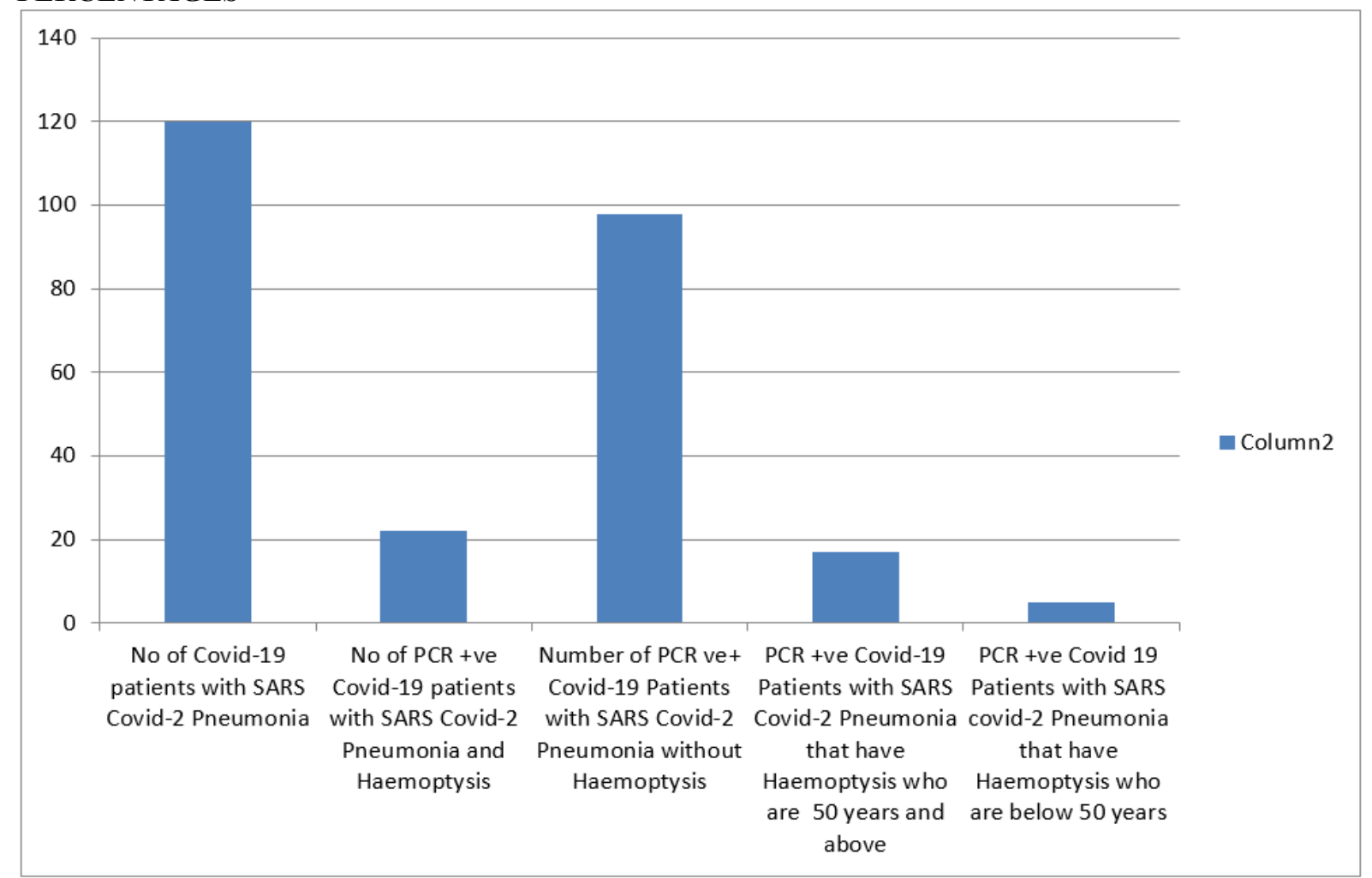

\section{FIGURE I}




\title{
PIE CHART SHOWING THE DISTRIBUTION OF SOME COVID-19 MEDICAL FEATURES WITH PERCENTAGES
}

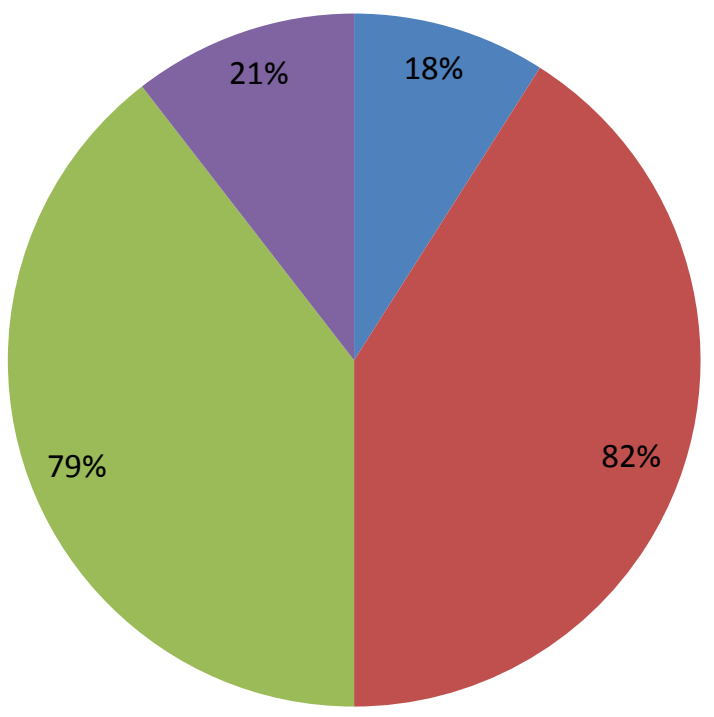

\author{
No of PCR +ve Covid-19 patients \\ with SARS Covid-2 Penumonia and \\ Haemoptysis \\ Number of PCR ve+ 98 Covid-19 \\ patients with SARS Covid-2 \\ Penumonia without Haemoptysis \\ PCR +ve Covid-19 Patients with \\ SARS Covid-2 Penumonia that \\ have Haemoptysis who are \\ 50years and above \\ PCR +ve Covid-19 Patients with \\ SARS Covid-2 Penumonia that \\ have Haemoptyiss who are below \\ 50 years.
}

\section{FIGURE II}

\section{DISCUSSION}

Viruses are a force of nature capable of affecting the biology of their host cells. While the microbial world in Endowed with certain proteins capable of inducing allergy, others affect haemostasis possibly through interaction with platelet factor 4 (PF4) that may precipitate clot formation and ultimately bleeding.

The results from this work show that $18 \%$ of the PCR +ve Covid-19 patients with SARS Covid-2 pneumonia were found to have associated haemoptysis, a feature possibly due to thrombo-embolic phenomenon. Covid-19 coagulopathy has made it to the medical literature and it has become a reference a phenomenon used in explaining some features of the disease like the clinically significant lowering of the percent Oxygen saturation measured in the invasively ventilated patients (Zagrillo et al 2020). The ensuring hypoxia may explain the extreme lethargy and debilitation that lead to deaths.

PCR + ve Covid-19 Patients with SARS Covid-2 Pneumonia that have Haemoptysis and are 50 years and above are represented as $79 \%$. This suggests that older age represent an increase vulnerability to development of haemoptsis in PCR +ve Covid-19 patients with SARS Covid-2 pneumonia. Pre-existing conditions in old age have been indicated in cases with Covid-19 as bad prognostic factor. With advancing age the immune functions are progressively less efficient and the tendency for allergies and other forms of biological over-reactities become even prevalent. It also translates to the fact that younger individual have less tendency to develop the like of cytokine storm and the thrombo-embolic phenomenon.

\section{CONCLUSION:}

The understanding of the pathophysiology of the PCR + ve SARS Covid-2 pneumonia is key to developing efficient management protocol and limiting the death rate in this pandemic. A broader understanding will add to preparedness for a possible future pandemic disease of infectious viral induced Flu with the like of the mortality like that of Covid-19. Multiple and more expanded studies may be enormous help.

\section{REFERENCES}

1. Martin J. Tobin, Franco Laghi, and Amal Jubran (2020). Why Covid-19 Silent Hypoxemia Is Baffling to PhysiciansAm J Respir Crit Care Med. 2020 Aug 1;202(3):356-360.

2. Zangrillo A, Baretta L, Scandroglio AM, et al. (2020). Characteristics, treatment, outcomes and cause of death of invasively ventilated patients with Covid-19 ARDS in Milan Italy. Crit Care Resusc.

3. Huang C, WangY, Li X, et al (2020). Clinical Features of Patients Infected with 2019 Novel Corona in Wuhan, China. Lancet. 2020;395:497-506. 